\title{
Small RNA expression from viruses, bacteria and human miRNAs in colon cancer tissue and its association with microsatellite instability and tumor location
}

\author{
Robin Mjelle ${ }^{1 *}$, Wenche Sjursen ${ }^{1,2}$, Liv Thommesen ${ }^{1,3}$, Pål Sætrom ${ }^{1,4,5,6}$ and Eva Hofsli ${ }^{1,7}$
}

\begin{abstract}
Background: MicroRNAs (miRNA) and other small RNAs are frequently dysregulated in cancer and are promising biomarkers for colon cancer. Here we profile human, virus and bacteria small RNAs in normal and tumor tissue from early stage colon cancer and correlate the expression with clinical parameters.

Methods: Small RNAs from colon cancer tissue and adjacent normal mucosa of 48 patients were sequenced using Illumina high-throughput sequencing. Clinical parameters were correlated with the small RNA expression data using linear models. We performed a meta-analysis by comparing publicly available small RNA sequencing datasets with our original sequencing data to confirm the main findings.

Results: We identified 331 differentially expressed miRNAs between tumor and normal samples. We found that the major changes in miRNA expression between left and right colon are due to miRNAs located within the Hoxdevelopmental genes, including miR-10b, miR-196b and miR-615. Further, we identified new miRNAs associated with microsatellite instability (MSI), including miR-335, miR-26 and miR-625. We performed a meta-analysis on all publicly available miRNA-seq datasets and identified 117 common miRNAs that were differentially expressed between tumor and normal tissue. The miRNAs miR-135b and miR-31 were the most significant upregulated miRNA in tumor across all datasets. The miRNA miR-133a was the most strongly downregulated miRNA in our dataset and also showed consistent downregulation in the other datasets. The miRNAs associated with MSI and tumor location in our data showed similar changes in the other datasets. Finally, we show that small RNAs from Epstein-Barr virus and Fusobacterium nucleatum are differentially expressed between tumor and normal adjacent tissue.
\end{abstract}

Conclusions: Small RNA profiling in colon cancer tissue revealed novel RNAs associated with MSI and tumor location. We show that Fusobacterium nucleatum are detectable at the RNA-level in colon tissue, and that both Fusobacterium nucleatum and Epstein-Barr virus separate tumor and normal tissue.

Keywords: miRNA, isomiR, ncRNA, sRNA, Epstein-Barr virus, Fusobacterium nucleatum, Colon cancer, High throughput sequencing

\footnotetext{
* Correspondence: robin.mjelle@ntnu.no

${ }^{1}$ Department of Clinical and Molecular Medicine, Norwegian University of

Science and Technology, NTNU, Erling Skjalgssons gt 1, 7030 Trondheim,

Norway

Full list of author information is available at the end of the article
}

(c) The Author(s). 2019 Open Access This article is distributed under the terms of the Creative Commons Attribution 4.0 International License (http://creativecommons.org/licenses/by/4.0/), which permits unrestricted use, distribution, and reproduction in any medium, provided you give appropriate credit to the original author(s) and the source, provide a link to the Creative Commons license, and indicate if changes were made. The Creative Commons Public Domain Dedication waiver (http://creativecommons.org/publicdomain/zero/1.0/) applies to the data made available in this article, unless otherwise stated. 


\section{Background}

Both host small RNAs (sRNAs) and bacterium and virus pathogens are implicated in the pathogenesis of several human cancers, including colorectal cancer (CRC). MicroRNAs (miRNAs), which is one of the most studied classes of sRNAs, have altered expression profiles in CRC and these profiles differ between CRC subtypes. For example, miRNAs correlate with microsatellite instability (MSI) status [1, 2], tumor location [3, 4], BRAF and KRAS mutation [5, 6] and tumor stage [7]. It is important to understand the underlying molecular mechanisms of CRC heterogeneity, since these mechanisms influence treatment decision-making.

Many studies have investigated the role of microorganisms in CRC, mostly focusing on the gut microbiota (e.g. $[8,9])$. Analyses by $16 \mathrm{~S}$ ribosomal DNA sequencing or real-time PCR have demonstrated enrichment of bacteria, including the oral bacterium Fusobacterium nucleatum ( $F$. nucleatum), in tumor compared to adjacent normal tissue [10-14]. F. nucleatum and other bacteria can modulate the microenvironment to cause inflammation and increase proliferation $[15,16]$.

In contrast to bacteria, the role of viral pathogens in CRC is less explored. Human Papilloma Virus (HPV) is detected in CRC tumors in some studies; however, no consensus is reached regarding its role, and the frequency of detection varies largely between studies [17]. The role of HPV is more evident for anal cancer, in which HPV is detected in the majority of the malignancies [18]. Epstein-Barr virus (EBV) is detected in CRC tumors, also with varying frequency [19-23]. Other viruses such as John Cunningham virus and Human Cytomegalovirus have been detected in CRC tissue and are suggested to have a role in enhancing cell proliferation [24, 25].

Here we use small RNA sequencing to analyze 48 early stage colon tumors and 48 matched adjacent normal controls. By combining our data with small RNA sequencing data from The Cancer Genome Atlas (TCGA) and three other cohorts [7, 26, 27], we provide a comprehensive view of canonical miRNAs, miRNA variants (isomiRs), and other small RNAs that differ between tumor and normal samples and between clinical subtypes of the tumors. Furthermore, we show that a large proportion of the sequencing reads that did not match any region in the human genome mapped to virus and bacterial species. Among the most prominent species were Fusobacterium nucleatum and EpsteinBarr-virus, which both showed over-expression in tumors compared with normal adjacent colon. Our results show that small RNAs can provide comprehensive and consistent data for early detection and subtyping of colon cancer.

\section{Methods}

\section{Patient samples}

The current study was performed as a retrospective study on samples from St. Olavs Hospital biobank. Samples from patients with newly diagnosed primary colon cancer from two hospitals in Norway (St Olavs Hospital, Trondheim and Hamar Hospital, Hamar) were included, as described in [28]. Tumor tissue from colon (coecum to sigmoideum, not rectum) and adjacent normal mucosa from 48 patients with tumors detected at early stages I-II (no lymph nodes or distant metastases), were included in the present study. All patients gave written consent to attend the study.

\section{Tumor molecular analyses and clinical parameters}

Status of MSI, BRAF mutation (V600E) and methylation of MLH1 promoter in tumor were analyzed as described [28]. KRAS mutation analyses were performed in DNA isolated from tumor tissue by sequencing of exon 2 and 3, to identify activating mutations in codon 12, 13 and 61 . Primers for exon 2 were 5'-TGGTGGAGTATTTGATAGTGT-3' (forward) and 5'-CCTCTATTGTTGGATCATATTC-3' (reverse) and primers for exon 3 were 5'-GGTGCACTG TAATAATCCAGACTGTGT-3' (forward) and 5'-TGCA TGGCATTAGCAAAGAC-3' (reverse).

Right-sided colon was defined as location 1-5 (appendix, coecum, ascendens and right flexure and transversum). Left-sided colon was defined as location 6-10 (left flexure, descendens, sigmoid, rectosigmoid and rectum).

\section{RNA isolation}

Total RNA was isolated from tissue samples by using miRVana RNA isolation kit (ThermoFisher). RIN values were measured using Eukaryote total RNA assay on the Agilent 2100. RIN >9 was regarded as high quality and sufficient for sequencing.

\section{Preparation of CDNA library for small RNA sequencing}

Small RNA sample preparation was performed using the TruSeq small RNA protocol (Illumina) according to the manufacturer's instructions, using $1 \mu \mathrm{g}$ RNA and 13 PCR cycles. During the 3' ligation step, 10 synthetic calibrator RNAs were added to the master mix to serve as internal controls. The miRNA fragments were sequenced on the Illumina HiSeq 2500 system using 50 base pair single read.

\section{Mutation count analysis}

Masked somatic mutations for TCGA colon adenocarcinomas were downloaded from the national cancer institute's genome data commons. A linear model correlating somatic mutation counts in exomes with presence of loss-of-function and missense mutations in one or more of the MMR genes MSH2, MSH6, MLH1, and 
PMS2 in TCGA were created using the $l m$ function in R in the following form: Count $\sim$ LossOfFunction + Missense. Here, "Count" is the $\log 2$ number of mutations, and "LossOfFunction" and "Missense" is presence of loss-of-function and missense mutation, respectively. The alternative model including miR-155 expression had the following form: Count $\sim$ LossOfFunction + Missense + miR155, where miR155 was $\log 2$ normalized miR-155-5p expression. The $p$-values represents the $\mathrm{p}$-values from the F-statistics in the linear model in $\mathrm{R}$.

\section{Reverse transcription quantitative PCR (RT-qPCR)}

We used the Taqman technology for RT-qPCR analyses (ThermoFisher). Custom probes were designed using Custom TaqMan ${ }^{\circ}$ Small RNA Assays. We used the following probes:

Fusobacterium probe 1: GGUCGCAUAGCUCAGUU GGGAGAGCACC.

Fusobacterium probe 2:ACCGGGAUGGACAAACC UCUGAUGUACCAGUU.

miR-BART10-3p: TACATAACCATGGAGTTGGCT. miR-BART19-5p. Cat. \#4427975. Assay ID: 006693.

\section{Results}

\section{Patient cohort}

Patient characteristics are described in Additional file 1: Table S1. Of the 48 patients, 23 were men and 25 were women. Median age at diagnosis was 76 years (range 30-93). 18 patients were diagnosed with MSI and 30 with MSS. 17 patients were characterized with V600E-BRAF mutation, 9 with KRAS mutation and 16 with MLH1 methylation. 14 patients were diagnosed with left-sided tumor and 34 with right-sided tumor.

\section{MicroRNAs and isomiRs are consistently differentially} expressed between tumors and normal colon across datasets Based on a cohort of 48 stage I-II colon cancer patients, we set out to characterize consistent expression differences in sRNAs between cancer and normal tissue, and between colon cancer subtypes characterized by MSI status, BRAF and KRAS mutations, and tumor location (Additional file 1: Table S1). Small RNA sequencing of the 96 paired tumor and normal samples identified miRNAs as the dominant class of RNAs, followed by transfer RNAs (tRNAs), small nucleolar RNAs (snoRNAs), ribosomal RNAs (rRNAs), and other sRNAs (Additional file 2: Sequencing Statistics, Additional file 3: Figure S1A-C).

Focusing on the miRNA expression data revealed clear separation of most tumor samples from the adjacent normal mucosa (Fig. 1a). 331 miRNAs were detected as differentially expressed between tumor and normal, which includes about $66 \%$ of all miRNAs after filtering out those with low expression (Additional file 1: Table S2). Of these, 88 miRNAs had an absolute log fold-change $(\operatorname{logFC})>1$ between tumor and normal (Fig. 1b). The miRNAs miR-135b-5p, miR-21-3p, miR-21-5p and miR-584-5p had higher expression in tumor tissue compared to normal tissue for all patients, making these miRNAs strong biomarker candidates for tumor tissue. Clustered miRNAs, such as the oncogenic miR-17-92 cluster, tended to have similar expression changes, suggesting that altered transcription explain many miRNA expression changes between tumor and normal (Additional file 4: Figure S2A, Additional file 3: Clustered miRNAs tend to be co-expressed).

For $48 \%$ of the detected miRNAs, the highest expressed miRNA sequence differed from the canonical miRNA sequence reported in miRBase, indicating that isomiRs are important to consider when analyzing miRNA expression (Additional file 4: Figure S2B). After filtering, we found 2451 of 3618 isomiRs (68\%) to be differentially expressed. The significant isomiRs derived from 343 unique miRNAs, with hsa-miR-192-5p having the highest number of significant isomiRs (131 isomiRs).

To validate our differentially expressed miRNAs we reanalyzed three publicly available datasets that contained small RNA sequencing data for normal and tumor CRC tissue: Neerincx [26], Sun [27], and Röhr [7]. We detected 225 and 202 differentially expressed miRNAs for the Neerincx and Sun data sets, respectively, and 117 of these miRNAs shared significant differential expression with our data (Fig. 1c, Additional file 2: Table S2). The expression changes for the significant miRNAs were highly correlated between these three datasets, with only a few miRNAs being regulated in opposite direction (Fig. 1d). Although we detected no significant miRNAs in Röhr (Additional file 2: Table S2), expression changes were highly correlated between the miRNAs in Röhr and our data (Fig. 1d). Similarly, isomiR expression changes were highly correlated (Additional file 4: Figure S2C), confirming consistent miRNA expression differences between tumor and normal tissue for all four datasets.

\section{Several sRNA classes are consistently differentially expressed between tumors and normal colon across datasets}

Having determined strong and consistent expression differences between tumor and normal tissue for miRNAs, we analyzed the other detected sRNAs. Of 18,735 unique non-miRNA sRNAs species, 363 were differentially expressed between tumor and normal tissue (Fig. 2a). Grouping the significant sRNAs by their class showed that small nuclear RNAs (snRNAs), rRNAs, signal recognition particle RNAs (SRPs), and other RNAs were upregulated in tumor cells (Additional file 5: Figure S3A). SnoRNAs was the only class downregulated in tumor cells, but this was largely due to several large snoRNA clusters being downregulated (Additional file 5: Figure S3B, C, 

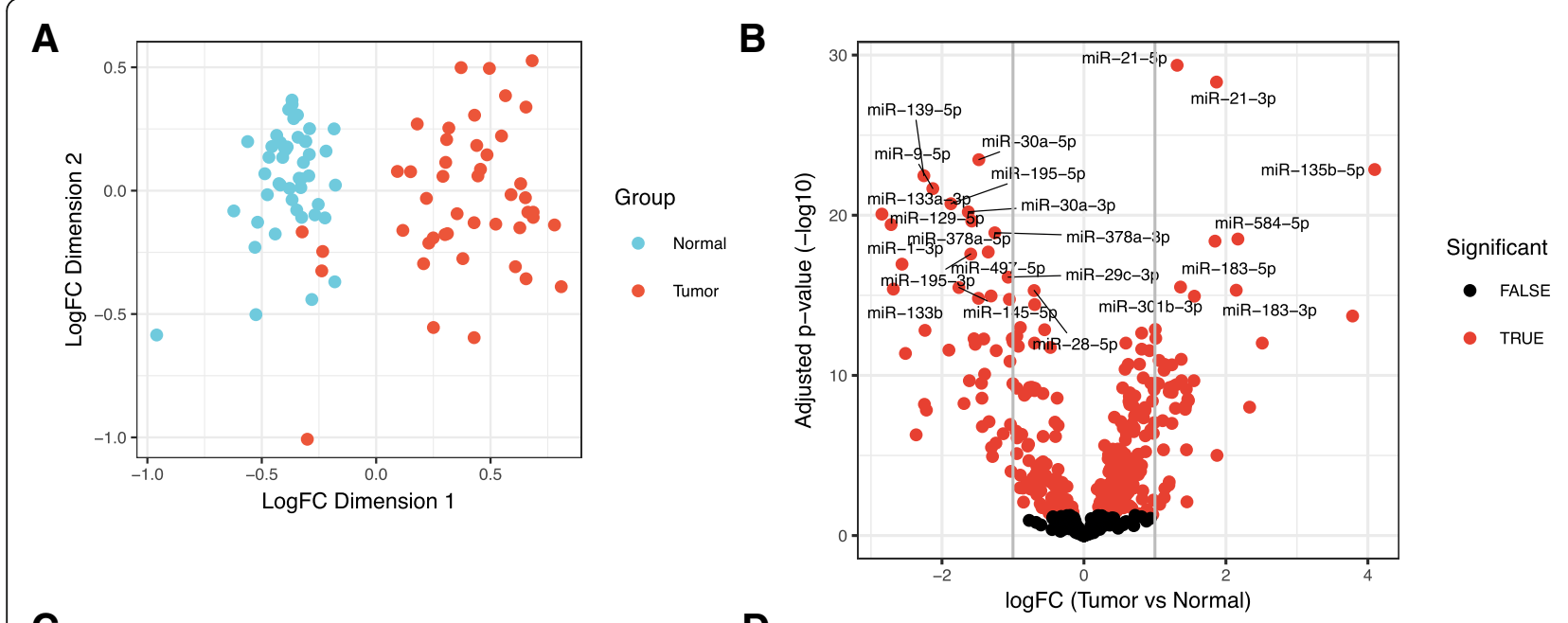

C

D

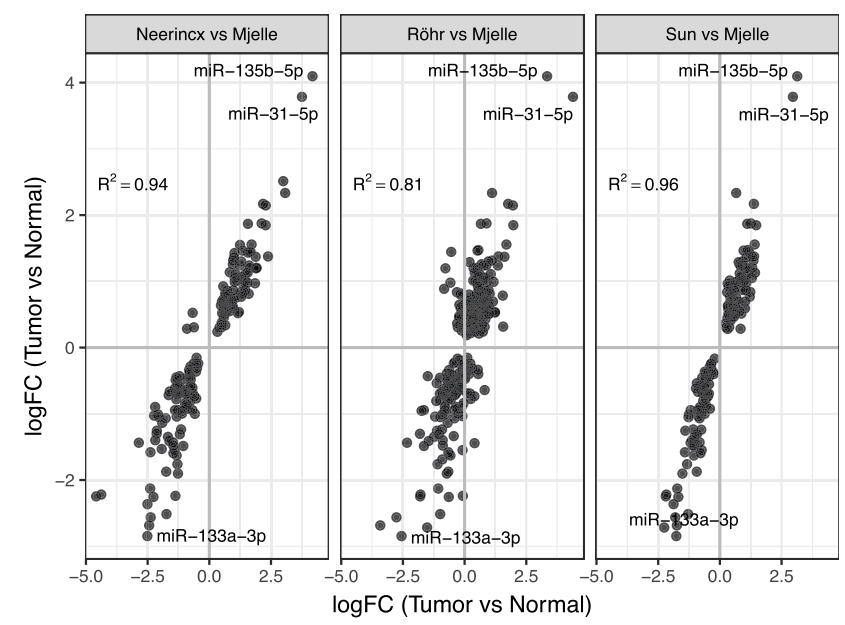

Fig. 1 MicroRNAs and isomiRs are consistently differentially expressed between tumors and normal colon. a Multidimensional scaling plot (MDS plot) of the canonical miRNAs. Tumor samples are depicted in turquoise and matched adjacent normal samples are depicted in red. $\mathbf{b}$ Volcano plot showing expression differences for individual miRNAs between tumor and normal. The $x$-axis shows fold change values (log2) between tumor and normal tissue. The statistical comparison used was tumor vs normal such that a positive fold change indicates that the miRNA is upregulated in tumor tissue compared to normal tissue. The $y$-axis shows the minus log10 adjusted $p$-value, such that a higher value corresponds to higher significance. Red dots indicate that the miRNA is significant with adjusted p-value less than 0.05. c Venn-diagram showing the number and overlap of significant miRNAs in our dataset and the datasets of Neerincx and Sun. $\mathbf{d}$ Scatterplot comparing fold-change values between datasets. The most significantly upregulated miRNAs are shown with names as well as miR-133 which is the miRNA with the lowest logFC in our data. The statistical comparison is described in B). The correlation value is the Pearson correlation between the logFC values in the two datasets that are being compared

Additional file 3: Clustered sRNAs). These clusters included two (ID12, ID13) located next to two miRNA clusters (ID2, ID3) and the MEG3 and MEG8 genes on chromosome 14 (Additional file 5: Figure S3D). Data from cap analysis of gene expression (CAGE) suggest that MEG3 is the host gene for miRNA clusters ID2 and ID3 [29], and low expression of $M E G 3$ is previously shown to be associated with poor survival in CRC [30].

Comparing expression differences for sRNAs between ours and the Neerincx, Sun, and Röhr datasets showed general positive but varying correlations for the different sRNAs classes (Fig. 2b). SnoRNAs and tRNAs were highly correlated for all datasets, whereas snRNAs only showed strong correlation to our results in the Neerincx data. As for miRNAs, the Röhr dataset had no significant sRNAs, but showed positive correlations to our results. Overall, snoRNAs, tRNAs, lncRNAs, and rRNAs showed consistent differential expression between tumor and normal colon.

\section{MicroRNA differences between right and left normal colon are reduced in tumors}

Next we asked whether miRNA expression was associated with available clinicopathological characteristics (tumor location and MSI status) for samples in our cohort (Additional file 2: Table S1), in Neerincx, and for 

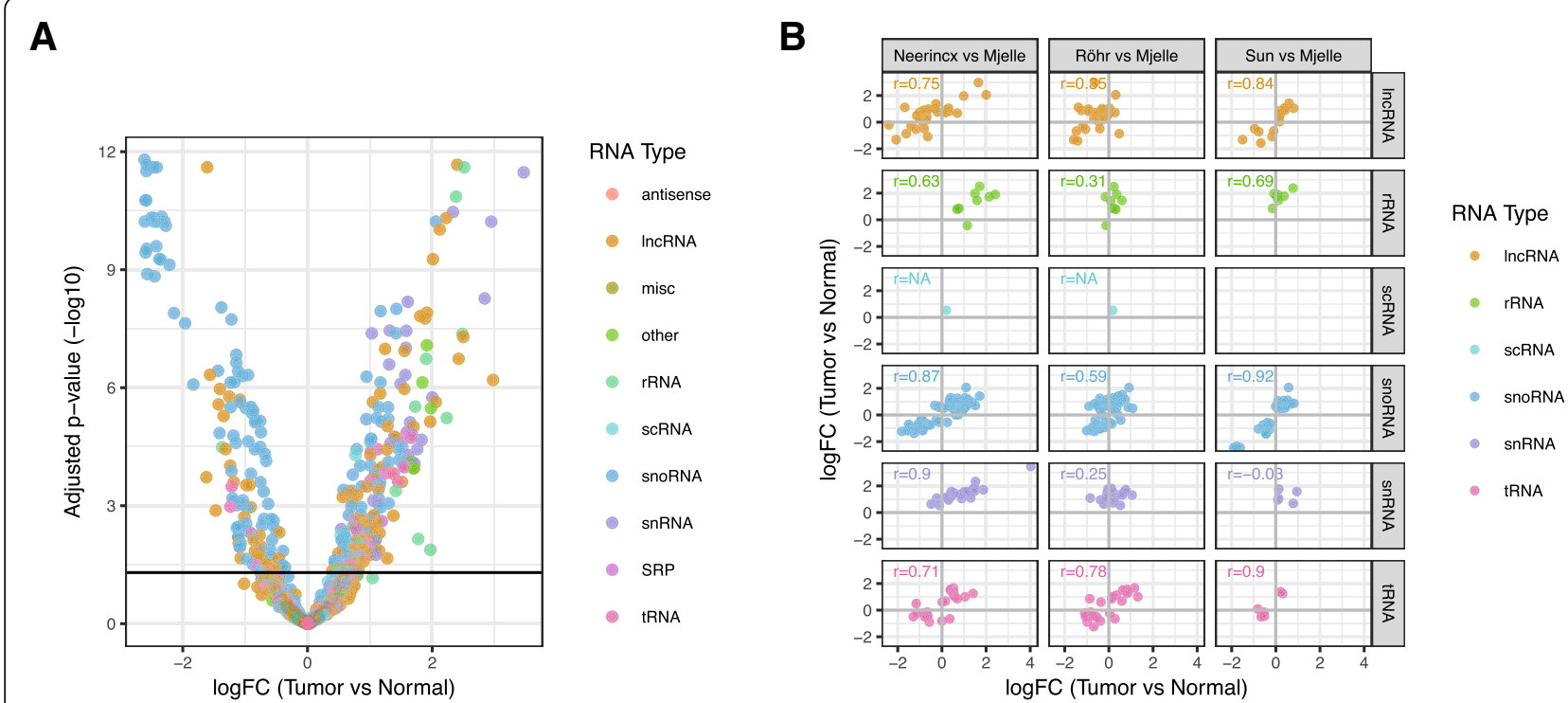

Fig. 2 Several sRNAs classes are consistently differentially expressed. a Volcano plot showing expression differences for individual ncRNAs between tumor and normal (see Fig. 1b). Each RNA type is shown with a unique color. b Scatterplots comparing fold-change values between datasets for different ncRNA types (see Fig. 1d)

colon cancer samples in The Cancer Genome Atlas (TCGA) [31]. CRC tumors are classified as right or left depending on colon localization. The right-sided colon is derived from the midgut and includes the proximal two-thirds of the transverse colon, ascending colon and caecum, whereas left-sided colon is derived from the hindgut and includes the distal third of the transverse colon, splenic flexure, descending colon, sigmoid colon and rectum [32]. It is often challenging to define tissue samples from the transversum region as part of the right or left colon. We therefore investigated the differences in miRNAs expression between right and left colon for the normal samples with location 5 defined as right colon or having samples with location 5 removed from

\section{A}

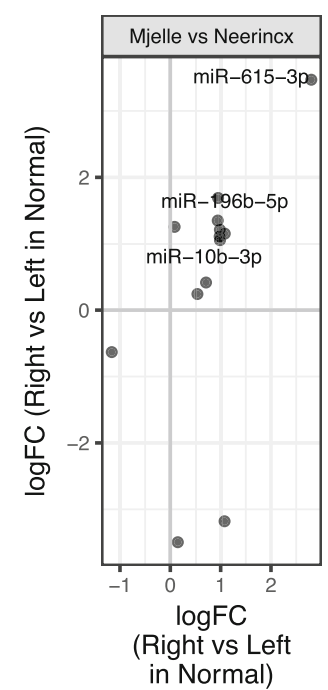

B

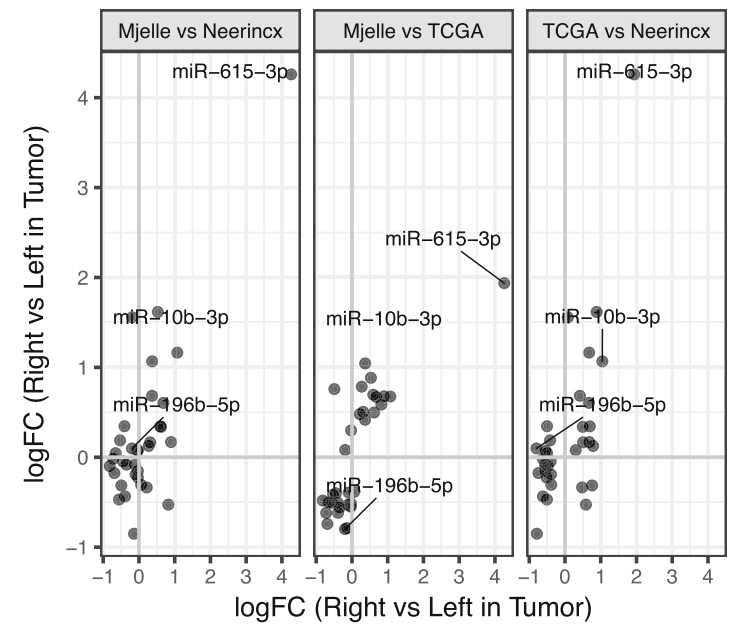

C

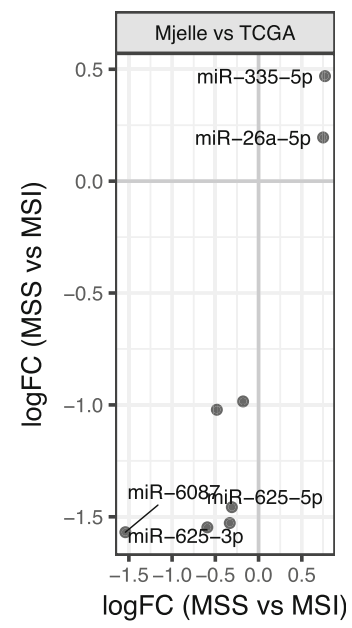

Fig. 3 Meta-analysis of miRNAs associated to clinical parameters. a, b MicroRNAs differentially expressed between right and left (a) normal colon and (b) tumor tissue in the Neerincx, TCGA, and our datasets. Positive fold changes indicate that the corresponding miRNA is upregulated in right compared to left colon. c MicroRNAs differentially expressed between MSI positive and negative tumors in the TCGA and our datasets. Positive fold changes indicate that the corresponding miRNA is upregulated in MSS compared to MSI 
the analysis. By including location 5 to the right colon samples, we detected 10 differentially expressed miRNAs (Fig. 3a; Additional file 1: Table S3). By exlcuding samples with location 5 from the analysis, we detected the same 10 miRNAs as differentially expressed, in addtion to miR-1224-5p, which was downregulated in right colon. This shows that in our data there is only minor differences in miRNA expression depending on whether transversum is defined as right colon or remove from the analysis. We therefore chose to include samples with location 5 to the right colon samples. For the normal samples, we found most of the significant miRNAs to be upregulated in right colon (Fig. 3a; Additional file 1: Table S3). When comparing with the other datasets, the miRNAs showed consistent expression, except for miR-490, which was highly downregulated in Neerincx and slightly upregulated in our dataset. Three of the miRNAs upregulated in right normal colon - miR-615, miR-10b and miR-196b - reside in homeobox (Hox) clusters $\mathrm{C}, \mathrm{D}$, and $\mathrm{A}$, respectively, indicating that some of the differences in miRNA expression could be due to differences in expression of Hox developmental genes (Additional file 5: Figure S3E). Differences in isomiR expression were also highly consistent between the two datasets (Additional file 6: Figure S4A). Next, we analyzed the tumor samples and compared right and left colon in our dataset and in Neerincx and TCGA, while adjusting for MSI status when available (not available in Neerincx). Overall, we observed consistent expression for most miRNAs and isomiRs across the datasets and especially high consistency between our dataset and TCGA (Fig. 3b; Additional file 6: Figure S4B; Additional file 1: Table S3).

Generally, different miRNAs were differentially expressed between right and left colon in cancer and normal tissue. MicroRNA miR-196b, for example, was highly upregulated in right normal tissue, but downregulated or unchanged in right vs left tumor tissue, indicating that this miRNA's expression depended on location and disease. Overall, only miR-615-3p and its isomiRs showed significant and consistent expression differences between left and right colon in both normal and tumor tissue (Additional file 6: Figure S4C, D).

\section{Tumor mutation levels is associated with miR-155-5p expression}

Moving on, we compared MSI positive and negative tumors in our dataset and TCGA, while adjusting for tumor location. Both miRNAs and isomiRs showed consistent expression differences in the two datasets (Fig. 3c; Additional file 6: Figure S4E, Additional file 1: Table S4), though only isomiRs for miR-26a-5p were significant in both datasets.
Microsatellite instability is associated with inactivation of DNA mismatch repair (MMR) genes causing increased mutation count in the cell. We therefore analyzed the association of loss-of-function or missense mutations in MMR genes and the number of somatic mutations in the TCGA dataset. Using linear modeling, we found that somatic loss-of-function or missense mutations in MMR genes were associated with approximately 8 and 4 times higher somatic mutation counts, respectively (estimated slopes of 3.02 and $2.01 ; p<2 \mathrm{e}-16$ and $p=2.85 \mathrm{e}-13$; model $\mathrm{r}^{2}=0.33$ ).

As miR-155-5p, which was significantly downregulated in MSI tumors, has been shown to target the MMR genes in colon cancer [33], we asked whether adding the expression of miR-155-5p in the abovementioned model could improve its predictions of somatic mutation counts. Indeed, this combined model explained an additional $8 \%$ of the variation in mutation counts and estimated that doubling miR-155-5p expression gave a 1.5 increase in somatic mutation counts (slope $0.6 ; p=3.25 \mathrm{e}-15$; model $\mathrm{r}^{2}=0.41$ ). Increased mutation count was associated with increased miR-155-5p expression only in tumors negative for loss-of-function or missense mutations in MMR genes (Additional file 6: Figure S4F, G), which is consistent with miR-155-5p only affecting mutation levels when its MMR target genes produce functional proteins.

\section{Expression of bacteria and virus RNAs in CRC tissue}

As both bacteria and viruses have been associated with CRC tissue, we hypothesized that the small RNA sequencing data could be used to detect expression of bacterial and viral small RNAs. We restricted the bacteria analysis to F. nucleatum only, due to its proposed role in $\mathrm{CRC}$, whereas the virus analysis included all known viral miRNAs.

Paired tumor vs normal analyses of the Neerincx, Sun, and our datasets detected significant over-representation of F. nucleatum in tumors for two of the three datasets (Fig. 4a). In the TCGA dataset, which included 8 normal and 455 tumor samples, we detected F. nucleatum all normal and tumor samples and observed a trend towards increased expression in tumors (Additional file 7: Figure S5A, B).

Similar analyses on viruses detected miRNAs from Epstein-Barr virus (EBV) as highly upregulated in tumor samples compared to paired normal samples in all three datasets (Fig. 4b). In TCGA, 4 normal and 349 tumor samples had detectable levels of EBV miRNAs (Additional file 7: Figure S5C). Most of the detected EBV miRNAs were located in the BamHI A rightward transcripts (BARTs) cluster within the EBV genome; the BART miRNAs comprised 345 of 355 viral isomiRs. In total, we detected 28 different viral miRNAs, 25 of which were from EBV and 3 which were from and Human 
A

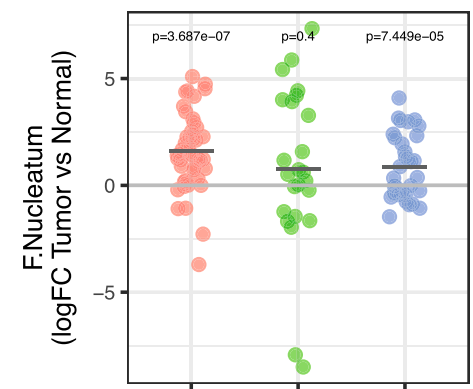

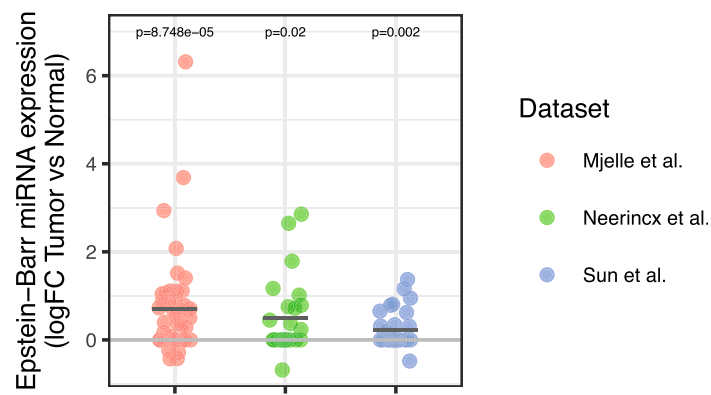

C

Dataset

- Mjelle et al.

Neerincx et al.

- Sun et al.
B

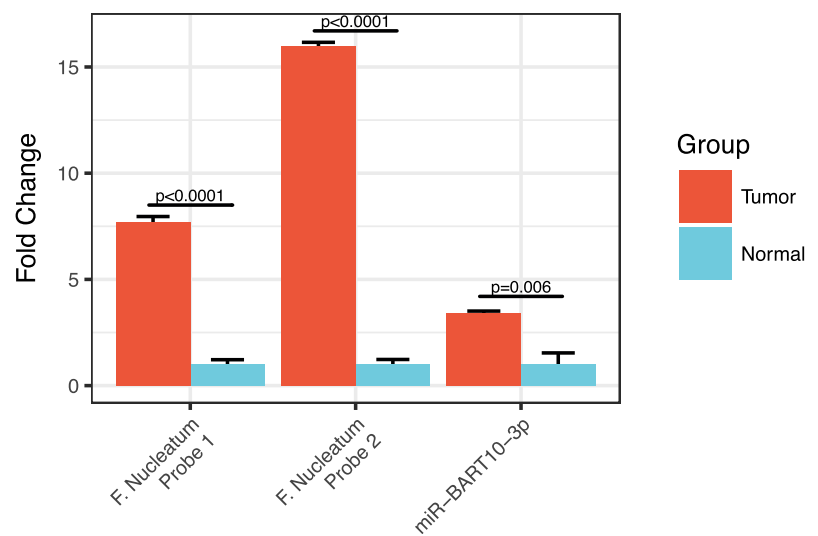

Fig. 4 Expression of bacteria and virus RNAs in CRC tissue. $\mathbf{a}$, b Fold-change values between paired tumor and normal tissue for (a) F. nucleatum and (b) Epstein-Barr virus in the Neerincx, Sun, and our datasets. c Bar-plot showing RT-qPCR validation of two probes against $F$. nucleatum and miR-BART10-5p. The normal samples are set to 1 and the error bars represent the standard deviation from three technical replicates. The experiment included 8 normal samples and 8 tumor samples

cytomegalovirus (HCMV). There was no correlation in tumor-expression of EBV miRNAs and F. nucleatum RNAs across the Neerincx, Sun, TCGA, and our datasets, indicating that presense of EBV and F. nucleatum in tumor tissue is independent (Additional file 7: Figure S5D).

We validated the expression differences by designing custom reverse transcription quantitative PCR (RT-qPCR) probes against two $F$. nucleatum RNAs and two EBV miRNAs, ebv-miR-BART10-3p and ebv-miR-BART19-5p and analyzing eight paired tumor and normal samples excluded from the sequencing experiment. The two F. nucleatum RNAs were highly upregulated in the tumor samples (Fig. 4c). MicroRNA miR-BART19-5p was undetected in 7 of the 8 normal samples and detected in all but one tumor sample ( $p=0.04$, McNemar's test), whereas miR-BART10-3p levels were significantly increased in the tumor samples (Fig. 4c). Furthermore, in situ hybridization against the $F$. nucleatum RNA in two additional patient samples revealed strong $F$. nucleatum signal in cells with malignant morphology and weaker staining in adjacent normal cells (Fig. 5).
Finally, we asked whether EBV and F. nucleatum expression were associated with clinical parameters in the Neerincx, TCGA, and our datasets. We found no associations for MSI and BRAF and inconsistent patterns for KRAS mutation and $F$. nucleatum, but tumor location for F. nucleatum and KRAS mutation for EBV showed consistent trends for all datasets and were significant in one dataset each (Additional file 7: Figure S5E-L).

\section{Discussion}

We have comprehensively analyzed miRNAs and sRNAs in early stage colon cancers and verified the major findings in our cohort by meta-analysis across multiple small RNA datasets.

MicroRNAs are known to be dysregulated in cancer and we find that much of this dysregulation is likely due to transcriptional effects on regions that contain several miRNAs in clusters. These include previously identified tumor suppressor (miR-154) and oncogenic (miR-17-92) loci $[34,35]$. Similarly, we find several clustered snoRNAs, which are co-transcribed as part of host primary 

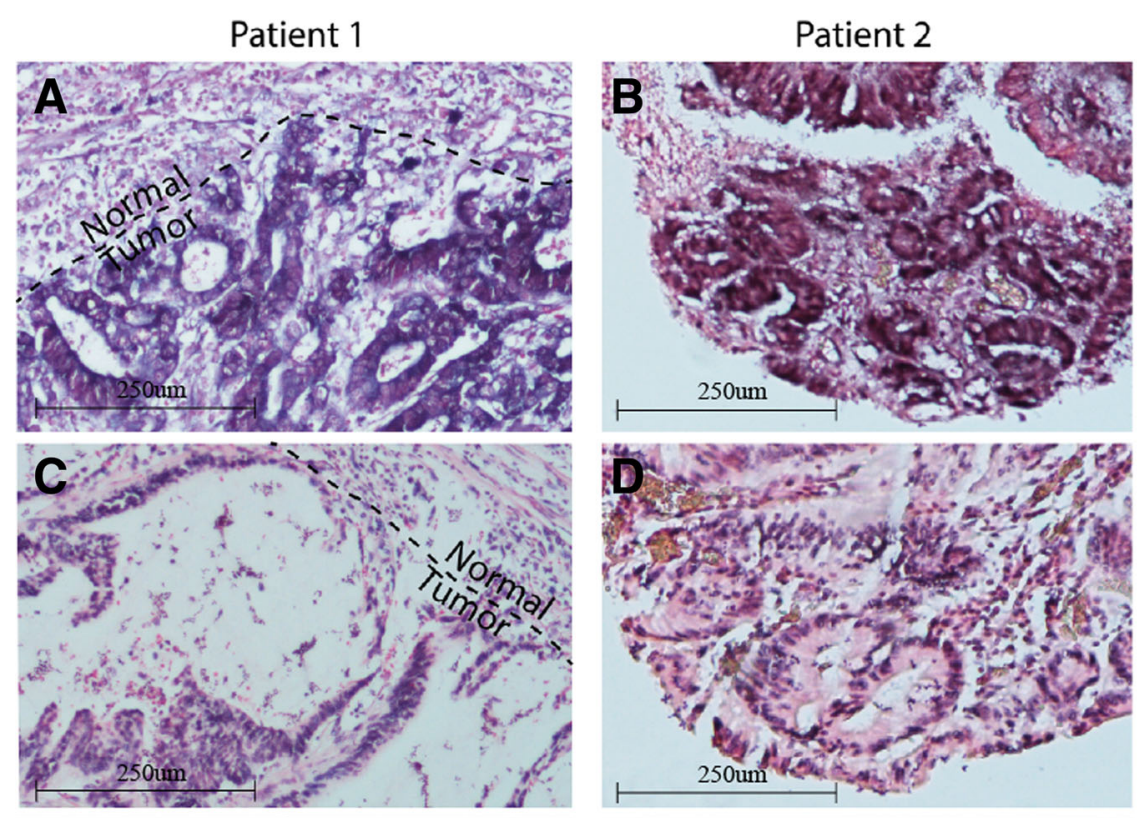

\section{$7 \pi$
2
$\frac{1}{2}$
$\frac{1}{2}$
$\frac{1}{3}$}
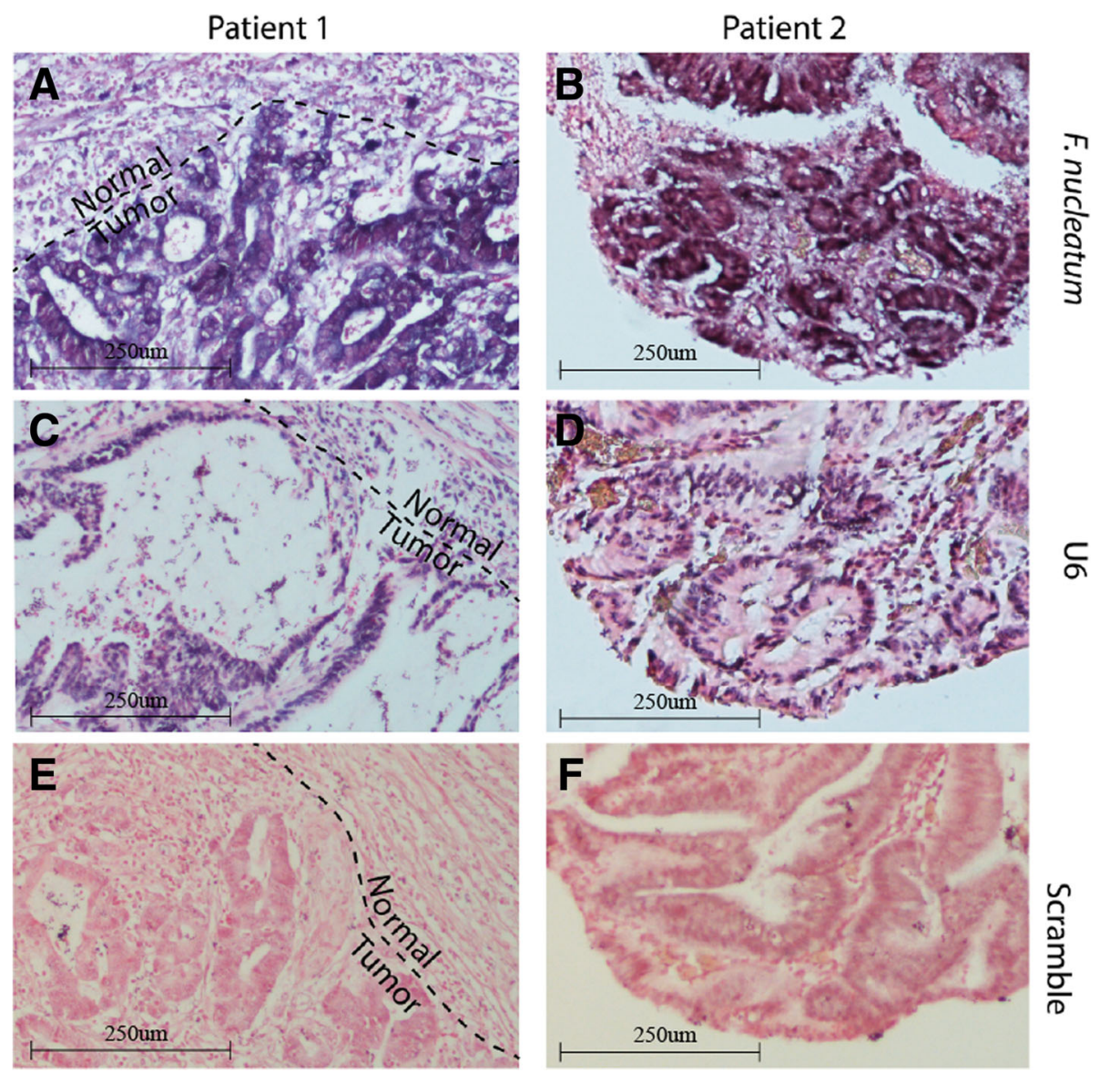

Fig. 5 In situ hybridization (ISH) of CRC formalin-fixed paraffin-embedded (FFPE) tissue from two patients (left and right). ISH staining of a small RNA fragment from (a, b) F. nucleatum, (c, d) U6 positive control, and (e, f) scramble negative control. For patient 1, part of the section included non-tumor cells, which is indicated by the dashed line

transcripts [36, 37], to be dysregulated. The growth arrest specific 5 (GAS5) long noncoding RNA hosts several snoRNAs [38], one of which (SNORD78, URS00006CFC33) is significantly upregulated in tumors in our study. GAS5 is implicated in a variety of cancers [39], and is shown to affect proliferation and to be a predictable prognostic marker in CRC patients [40]. In contrast to snoRNAs, the distinct upregulation of snRNAs in tumors cannot be explained by dysregulation of a common host gene, as these RNAs are located in different genomic regions. Interestingly, the tri-small ribonucleoprotein (tri-snRNP) spliceosome complex that binds snRNAs is commonly over-expressed in human primary and metastatic colon cancer [41]. This could explain why snRNAs are generally upregulated in tumor tissue.

We investigated if miRNA expression correlated with clinical parameters and if miRNAs could predict tumor subtypes. Consistent with a previous study [3], we found that miR-615-3p is highly upregulated in right-sided tumors compared to left-sided tumors. When examining the normal tissue, however, we also found miR-615-3p to be highly upregulated in right compared to left colon, suggesting that the expression difference between leftand right-sided tumors for miR-615-3p is due to underlying biological differences between left and right colon. Indeed, we found a larger set of miRNAs that separates left and right colon than separates left- and right-sided tumors. These include miR-196b and miR-10b, which are upregulated in right normal colon and, along with mir-615, are located within or in close proximity to the homeobox genes. The expression of these miRNAs is known to depend on these homeobox genes $[42,43]$. The homeobox genes play a role in gut development [44], and because the right and left colon develop from the hindgut and midgut, respectively, differences in transcription patterns between the two regions could explain some of the region-related expression differences for these miRNAs. Notably, miR-196b, which was upregulated in right normal colon, was downregulated or unchanged in right tumor colon, indicating that additional mechanisms affect miR-196b expression in tumors. 
Microsatellite instability is an important clinicopathological characteristic that determines treatment procedure and has prognostic value [45]. For instance, several recent studies have shown that MSI tumors respond well to immune therapy $[46,47]$ and have a nonbeneficial effect of adjuvant 5-FU-based chemotherapy [48]. We observed several miRNAs and isomiRs to be differentially expressed between MSI and MSS tumors - both in our and the TCGA cohort. Although few of these miRNAs were significant in both cohorts, the estimated expression differences for these miRNAs were highly reproducible (Fig. 3c). This high reproducibility suggests that the detected miRNAs do represent biological differences between MSI and MSS tumors. Indeed, whereas there was little overlap between significant MSI-related miRNAs in our and previous studies [2, 49-52], Earle et al., Lanza et al., Tang et al. and we detected miR-155 as upregulated in MSI tumors. Over-expression of miR-155 downregulates core DNA mismatch repair (MMR) genes and affects their protein expression [33]. We found that increased miR-155 levels correlate with increased mutation counts in tumors with intact MMR genes, suggesting a model where upregulation of miR-155 gives an increased mutational burden, possibly resulting in mutated MMR genes and MSI.

Bacteria have been associated with colorectal cancer, particularly the composition of the fecal and colon microbiome [53]. Sequencing of long RNAs and 16S DNA has detected $F$. nucleatum in tumor and normal colon tissue $[11,13]$. Moreover, F. nucleatum is present in metastatic CRC tissue, indicating that the bacterium is transferred during metastasis. We show that small RNAs of $F$. nucleatum are present in colon tissue and that the expression is higher in tumor than in normal tissue. Bacterial small RNAs have been detected in other bacteria species and have a variety of regulatory mechanisms [54]. It remains to be investigated whether small RNAs from $F$. nucleatum possess regulatory properties that could affect tumor development. Several studies have detected viral DNA in colon cancer tumors, the most common types being Human papillomaviruses (HPV), Human polyomaviruses and Human herpesviruses [55]. Many of these viruses encode proteins that could potentially be oncogenic and affect proliferation of host cells [56]. The Human herpesvirus EBV contain viral miRNAs, most of which are encoded from the BART loci. Several of these miRNAs are shown to target host mRNAs and some EBV-miRNAs target genes involved in cancer development $[57,58]$. Little is known about EBV-miRNAs in colon cancer, however, miR-BART19-3p is shown to target WIF1 [59], a gene important in colon cancer [60], and miR-BART1 is shown to target PSAT1 [61], a gene shown to promote proliferation of tumor cells [62]. However, none of these interactions have been shown in colon cancer cells. The EBV-miRNA miR-BART19-5p, which we found elevated in tumor tissue, is shown to target its own gene LMP1 [63], which is a functional homologue of tumor necrosis factor [64] that can downregulate host miR-146a. The EBV-miRNA miR-BART10-3p, which we found elevated in tumor tissue was previously found to directly targets $B T R C$, a gene found to be upregulated in colon cancer [65]. In summary, viral miRNAs could have significant impact on genes and cellular processes in the host and these interactions need further investigation, in particular in colon cancer.

\section{Conclusions}

Small RNA profiling in colon cancer tissue revealed unique expression patterns in comparison with adjacent normal tissue. We detected several miRNAs that correlated with MSI and tumor location and show that miR-155 expression together with MMR deficiency explain much of the MSI-associated mutations in colon cancer. We show for the first time that small RNAs from Fusobacterium nucleatum are present in colon tissue, and that both Fusobacterium nucleatum and Epstein-Barr virus separate tumor and normal tissue.

\section{Additional files}

Additional file 1: Table S1-S4. Differentially expression analysis and patient's characteristics. (DOCX $138 \mathrm{~kb}$ )

Additional file 2: Contains sequencing statistics results and additional methods description. (DOCX 49 kb)

Additional file 3: Figure S1. Sequencing statistics. A) Library size: Number of raw reads in the samples; Alignment: Number of reads that aligned to only one position in the genome (SingleAligned), multiple positions (MultiAligned) or not aligned (NotAligned); Features: Number of reads that aligned to miRBase, RNACentral database and to the calibrator RNAs; RNAs: Number of reads for the main RNA classes. B) Composition of RNAs in the samples shown as ratios. $\mathbf{C}$ ) The sequences of the calibrator RNAs. (PDF $492 \mathrm{~kb}$ )

Additional file 4: Figure S2. MicroRNA clusters and isomiRs. A) A scatterplot of genomic clustered miRNAs showing log fold change values between tumor and normal (y-axis) for each individual miRNA within the clusters. The clusters are indicated with a unique ID on each facet of the plot. The figure only includes miRNAs that are significantly differentially expressed between tumor and normal. B) Illustrations of the main types of isomiRs analyzed in the current study, exemplified for hsa-miR-10b. C) Scatterplot comparing fold-change values for isomiRs between our dataset and the Neerincx, Röhr, and Sun datasets. (PDF $1751 \mathrm{~kb}$ )

Additional file 5: Figure S3. Classes of ncRNAs and snoRNA clusters. A) Boxplot showing expression of the sRNAs that differ significantly between tumor and normal samples, grouped by RNA classes. B) A scatterplot of genomic clustered snoRNAs showing log fold change values between tumor and normal (y-axis) for each individual snoRNA within the clusters. The clusters are indicated with a unique ID on each facet of the plot. The figure only includes snoRNAs that are significantly differentially expressed between tumor and normal. The $p$-values are calculated using a two-tailed student's t-test. C) Log fold change values for snoRNAs that are not contained within a genomic cluster. D) Genome browser graphics of snoRNAs adjacent to the MEG3 gene. The graphics is from http://genome-euro.ucsc.edu. The miRNA clusters ID2 and ID3 are indicated with a red line below the graphics. E) Genome browser 
graphics of miR-10b, miR-615 and miR-196b and the adjacent RefSeq genes. (PDF $673 \mathrm{~kb}$ )

Additional file 6: Figure S4. MicroRNAs and isomiRs correlated with clinicopathological characteristics. A) Differentially expressed isomiRs in our dataset and the dataset of Neerincx et al. between right and left normal colon tissue. The comparison is Right-Left such that a positive fold change indicates that the corresponding miRNA is upregulated in right normal colon compared to left normal colon. B) Differentially expressed isomiRs in our data and the dataset of Neerincx et al. and TCGA comparing right and left tumor colon tissue. The statistical comparison is described in A). C) Differentially expressed miRNAs between right and left tumor tissue ( $x$-axis) compared with differentially expressed miRNAs between right and left normal tissue (y-axis), for the datasets Mjelle et al. and Neerincx et al. D) Similar comparison as in C), for isomiRs. E) Differentially expressed isomiRs in our data and the dataset and TCGA comparing MSS and MSI. The comparison is MSS-MSI such that a positive fold change indicates that the corresponding isomiR is upregulated in MSS compared to MSI. F-G) Mutation counts for tumors grouped by miR-155 expression level and presence of somatic (F) loss-of-function or (G) missense mutations in MMR genes. The mean value is indicated with a cross. The $p$-values is the output from the linear model. (PDF $1150 \mathrm{~kb}$ )

Additional file 7: Figure S5. Expression of F. nucleatum and EBV miRNA in TCGA data and correlation with clinical parameters across data sets. The p-values in Fig. S5E-L are calculated using an unpaired two-tailed Student's t-test. A) Expression of $F$. nucleatum in TCGA small RNA data. Left: All tumor samples compared to the eight normal samples. Right: The 8 paired tumor and normal samples. The $p$-values are calculated using an unpaired and paired two-tailed Student's t-test for the left and right boxplot, respectively. B) Expression of $F$. nucleatum in TCGA colon samples. C) Expression of EBV miRNAs in TCGA colon samples. The figure shows the sum of all detected EBV miRNA for each sample as log2 and is normalized based on library sizes of mature miRNAs in the corresponding samples. D) Correlation between $F$. nucleatum and EBV miRNA in Neerincx et al., Sun et al., TCGA, and our datasets. The correlation is calculated using the cor0 function in $\mathrm{R}$ with pearson correlation. E) F. nucleatum expression in MSI and MSS tumors in Mjelle et al. and TCGA. F) F. nucleatum expression in BRAF mutated and BRAF wild type (non-mutated) tumors in Mjelle et al. G) F. nucleatum expression in KRAS mutated and KRAS wild-type (non-mutated) tumors in Mjelle et al. and TCGA. H) F. nucleatum expression in right-and left-sided tumor tissue in Mjelle et al., Neerincx et al. and TCGA. I) EBV miRNA expression in right-and left-sided tumor tissue in Mjelle et al., Neerincx et al. and TCGA. J) EBV miRNA in BRAF mutated and BRAF wild type (non-mutated) tumors in Mjelle et al. K) EBV miRNA expression in MSI and MSS tumors in Mjelle et al. and TCGA. L) EBV miRNA expression in KRAS mutated and KRAS wild-type (non-mutated) tumors in Mjelle et al. and TCGA. (PDF $462 \mathrm{~kb}$ )

\section{Abbrevations}

BARTs: BamHI A rightward transcripts; CRC: colorectal cancer; F. nucleatum: Fusobacterium nucleatum; Hox: Homeobox; HPV: Human Papilloma Virus; IncRNAs: long non-coding RNAs; logFC: log fold-change; miRNA: microRNA; MMR: DNA mismatch repair; MSI: microsatellite instability; PCR EBV: Epstein-Barr virus; RT-qPCR: Reverse transcription quantitative PCR; snoRNAs: small nucleolar RNAs; snRNAs: small nuclear RNA; sRNAs: small RNAs; SRPs: signal recognition particle RNAs; TCGA: The Cancer Genome Atlas

\section{Acknowledgements}

We thank the Genomics Core Facility (GCF) for sequencing the samples and quality analysis. We thank Linn-Karina Myrland Selvik for isolating RNA. We thank Torild Gladsø and the people at the pathology department at St. Olavs Hospital for support on the ISH experiments.

\section{Funding}

The study was funded by a grant from Felles forskningsutvalg (FFU) at St. Olavs Hospital, Trondheim, Norway. RNA isolation and small RNA sequencing was funded by this grant.

\section{Availability of data and materials}

The sequencing data will be submitted to Sequence Read Archive (SRA) upon approval from the Regional Committees for Medical and Health Research Ethics (REK-midt). Count matrices are available by contacting the corresponding author

\section{Authors' contributions}

RM: Preparing the manuscript, data analysis, and laboratory work. PS: Data analysis and statistics, preparing the manuscript; WS: Study design, collecting patient characteristics and sample material, preparing the manuscript; LT: Study design, preparing the manuscript; EH: Preparing the manuscript, study design. All authors read and approved the final manuscript.

\section{Ethics approval and consent to participate}

The project is approved by the Regional Committees for Medical and Health Research Ethics (REK-midt) with the following reference number: 2010/955. Study participants gave informed consent in accordance with the Declaration of Helsinki.

\section{Consent for publication}

Not applicable.

\section{Competing interests}

The authors declare that they have no competing interests.

\section{Publisher's Note}

Springer Nature remains neutral with regard to jurisdictional claims in published maps and institutional affiliations.

\section{Author details}

${ }^{1}$ Department of Clinical and Molecular Medicine, Norwegian University of Science and Technology, NTNU, Erling Skjalgssons gt 1, 7030 Trondheim, Norway. ${ }^{2}$ Department of Medical Genetics, St Olavs Hospital, Trondheim Norway, Erling Skjalgssons gt 1, 7030 Trondheim, Norway. ${ }^{3}$ Department of Biomedical Laboratory Science, Norwegian University of Science and Technology, NTNU, 7491 Trondheim, Norway. ${ }^{4}$ Department of Computer and Information Science, Norwegian University of Science and Technology, NTNU, Sem Sælandsvei 9, 7491 Trondheim, Norway. ${ }^{5}$ Bioinformatics core facility-BioCore, Norwegian University of Science and Technology, NTNU, 7491 Trondheim, Norway. ${ }^{6}$ K.G. Jebsen Center for Genetic Epidemiology, Norwegian University of Science and Technology, NTNU, 7491 Trondheim, Norway. ${ }^{7}$ The Cancer Clinic, St. Olav's Hospital, Trondheim University Hospital, Trondheim, Norway.

Received: 8 October 2018 Accepted: 29 January 2019

Published online: 20 February 2019

\section{References}

1. Yamamoto H, Imai K. Microsatellite instability: an update. Arch Toxicol. 2015: 89(6):899-921.

2. Slattery ML, Wolff E, Hoffman MD, Pellatt DF, Milash B, Wolff RK. MicroRNAs and colon and rectal cancer: differential expression by tumor location and subtype. Genes Chromosom Cancer. 2011;50(3):196-206.

3. Schee K, Lorenz S, Worren MM, Gunther CC, Holden M, Hovig E, et al. Deep sequencing the MicroRNA transcriptome in colorectal Cancer. PLoS One. 2013;8(6):e66165.

4. Shao S, Neely BA, Kao TC, Eckhaus J, Bourgeois J, Brooks J, et al. Proteomic profiling of serial Prediagnostic serum samples for early detection of Colon Cancer in the U.S Military. Cancer Epidemiol Biomarkers Prev. 2017;26(5):711-8.

5. Slattery ML, Herrick JS, Mullany LE, Wolff E, Hoffman MD, Pellatt DF, et al. Colorectal tumor molecular phenotype and miRNA: expression profiles and prognosis. Mod Pathol. 2016;29(8):915-27.

6. Nosho K, Igarashi H, Nojima M, Ito M, Maruyama R, Yoshii S, et al. Association of microRNA-31 with BRAF mutation, colorectal cancer survival and serrated pathway. Carcinogenesis. 2014;35(4):776-83.

7. Rohr C, Kerick M, Fischer A, Kuhn A, Kashofer K, Timmermann B, et al. Highthroughput miRNA and mRNA sequencing of paired colorectal normal, tumor and metastasis tissues and bioinformatic modeling of miRNA-1 therapeutic applications. PLoS One. 2013;8(7):e67461. 
8. Gao R, Gao Z, Huang L, Qin H. Gut microbiota and colorectal cancer. Eur J Clin Microbiol Infect Dis. 2017;36(5):757-69.

9. Jobin C. Human intestinal microbiota and colorectal Cancer: moving beyond associative studies. Gastroenterology. 2017;153(6):1475-8.

10. Zhou Y, He H, Xu H, Li Y, Li Z, Du Y, et al. Association of oncogenic bacteria with colorectal cancer in South China. Oncotarget. 2016;7(49):80794-802.

11. Castellarin M, Warren RL, Freeman JD, Dreolini L, Krzywinski M, Strauss J, et al. Fusobacterium nucleatum infection is prevalent in human colorectal carcinoma. Genome Res. 2012;22(2):299-306.

12. McCoy AN, Araujo-Perez F, Azcarate-Peril A, Yeh JJ, Sandler RS, Keku TO. Fusobacterium is associated with colorectal adenomas. PLoS One. 2013;8(1): e53653.

13. Kostic AD, Gevers D, Pedamallu CS, Michaud M, Duke F, Earl AM, et al. Genomic analysis identifies association of fusobacterium with colorectal carcinoma. Genome Res. 2012:22(2):292-8

14. Li YY, Ge QX, Cao J, Zhou YJ, Du YL, Shen B, et al. Association of Fusobacterium nucleatum infection with colorectal cancer in Chinese patients. World J Gastroenterol. 2016;22(11):3227-33.

15. Kostic AD, Chun E, Robertson L, Glickman JN, Gallini CA, Michaud M, et al. Fusobacterium nucleatum potentiates intestinal tumorigenesis and modulates the tumor-immune microenvironment. Cell Host Microbe. 2013;14(2):207-15.

16. Newman JV, Kosaka T, Sheppard BJ, Fox JG, Schauer DB. Bacterial infection promotes colon tumorigenesis in Apc(min/+) mice. J Infect Dis. 2001;184(2): 227-30.

17. Lorenzon L, Ferri M, Pilozzi E, Torrisi MR, Ziparo V, French D. Human papillomavirus and colorectal cancer: evidences and pitfalls of published literature. Int J Color Dis. 2011;26(2):135-42.

18. Bucchi D, Stracci F, Buonora N, Masanotti G. Human papillomavirus and gastrointestinal cancer: a review. World J Gastroenterol. 2016;22(33):7415-30.

19. Song $L B$, Zhang $X$, Zhang $C Q$, Zhang $Y$, Pan ZZ, Liao WT, et al. Infection of Epstein-Barr virus in colorectal cancer in Chinese. Ai Zheng. 2006;25(11): 1356-60.

20. Al-Antary N, Farghaly H, Aboulkassim T, Yasmeen A, Akil N, Al Moustafa AE, Epstein-Barr virus and its association with Fascin expression in colorectal cancers in the Syrian population: a tissue microarray study. Hum Vaccin Immunother. 2017;13(7):1573-8.

21. Tafvizi F, Fard ZT, Assareh R. Epstein-Barr virus DNA in colorectal carcinoma in Iranian patients. Pol J Pathol. 2015;66(2):154-60.

22. Mehrabani-Khasraghi S, Ameli M, Khalily F. Demonstration of herpes simplex virus, cytomegalovirus, and Epstein-Barr virus in colorectal Cancer. Iran Biomed J. 2016;20(5):302-6.

23. Fiorina L, Ricotti M, Vanoli A, Luinetti $\mathrm{O}$, Dallera $\mathrm{E}$, Riboni R, et al. Systematic analysis of human oncogenic viruses in colon cancer revealed EBV latency in lymphoid infiltrates. Infect Agent Cancer. 2014;9:18.

24. Teo WH, Chen HP, Huang JC, Chan YJ. Human cytomegalovirus infection enhances cell proliferation, migration and upregulation of EMT markers in colorectal cancer-derived stem cell-like cells. Int J Oncol. 2017;51(5):1415-26.

25. Mou X, Chen L, Liu F, Lin J, Diao P, Wang H, et al. Prevalence of JC virus in Chinese patients with colorectal cancer. PLoS One. 2012;7(5):e35900.

26. Neerincx M, Sie DL, van de Wiel MA, van Grieken NC, Burggraaf JD, Dekker $H$, et al. MiR expression profiles of paired primary colorectal cancer and metastases by next-generation sequencing. Oncogene. 2015;4:e170.

27. Sun G, Cheng YW, Lai L, Huang TC, Wang J, Wu X, et al. Signature miRNAs in colorectal cancers were revealed using a bias reduction small RNA deep sequencing protocol. Oncotarget. 2016;7(4):3857-72.

28. Trano G, Sjursen W, Wasmuth HH, Hofsli E, Vatten LJ. Performance of clinical guidelines compared with molecular tumour screening methods in identifying possible lynch syndrome among colorectal cancer patients: a Norwegian population-based study. Br J Cancer. 2010;102(3):482-8.

29. de Rie D, Abugessaisa I, Alam T, Arner E, Arner P, Ashoor H, et al. An integrated expression atlas of miRNAs and their promoters in human and mouse. Nat Biotechnol. 2017;35(9):872-8.

30. Yin DD, Liu ZJ, Zhang E, Kong R, Zhang ZH, Guo RH. Decreased expression of long noncoding RNA MEG3 affects cell proliferation and predicts a poor prognosis in patients with colorectal cancer. Tumour Biol. 2015;36(6):4851-9.

31. Grossman RL, Heath AP, Ferretti V, Varmus HE, Lowy DR, Kibbe WA, et al. Toward a shared vision for Cancer genomic data. N Engl J Med. 2016;375(12):1109-12.

32. Stintzing S, Tejpar S, Gibbs P, Thiebach L, Lenz HJ. Understanding the role of primary tumour localisation in colorectal cancer treatment and outcomes. Eur J Cancer. 2017;84:69-80.
33. Valeri N, Gasparini P, Fabbri M, Braconi C, Veronese A, Lovat F, et al. Modulation of mismatch repair and genomic stability by miR-155. Proc Nat Acad Sci U S A. 2010;107(15):6982-7.

34. Laddha SV, Nayak S, Paul D, Reddy R, Sharma C, Jha P, et al. Genome-wide analysis reveals downregulation of miR-379/miR-656 cluster in human cancers. Biol Direct. 2013;8:10.

35. Hayashita Y, Osada H, Tatematsu Y, Yamada H, Yanagisawa K, Tomida S, et al. A polycistronic microRNA cluster, miR-17-92, is overexpressed in human lung cancers and enhances cell proliferation. Cancer Res. 2005; 65(21):9628-32.

36. Lestrade L, Weber MJ. snoRNA-LBME-db, a comprehensive database of human $\mathrm{H}$ / ACA and C/D box snoRNAs. Nucleic Acids Res. 2006;34(Database issue):D158-62.

37. Kiss-Laszlo Z, Henry Y, Bachellerie JP, Caizergues-Ferrer M, Kiss T. Site-specific ribose methylation of preribosomal RNA: a novel function for small nucleolar RNAs. Cell. 1996;85(7):1077-88.

38. Williams GT, Farzaneh F. Are snoRNAs and snoRNA host genes new players in cancer? Nat Rev Cancer. 2012;12(2):84-8.

39. Ma C, Shi X, Zhu Q, Li Q, Liu Y, Yao Y, et al. The growth arrest-specific transcript 5 (GAS5): a pivotal tumor suppressor long noncoding RNA in human cancers. Tumour Biol. 2016;37(2):1437-44.

40. Yin D, He X, Zhang E, Kong R, De W, Zhang Z. Long noncoding RNA GAS5 affects cell proliferation and predicts a poor prognosis in patients with colorectal cancer. Med Oncol. 2014;31(11):253.

41. Adler AS, McCleland ML, Yee S, Yaylaoglu M, Hussain S, Cosino E, et al. An integrative analysis of colon cancer identifies an essential function for PRPF6 in tumor growth. Genes Dev. 2014;28(10):1068-84.

42. Yekta S, Tabin CJ, Bartel DP. MicroRNAs in the Hox network: an apparent link to posterior prevalence. Nat Rev Genet. 2008;9(10):789-96.

43. Woltering JM, Durston AJ. MiR-10 represses HoxB1a and HoxB3a in zebrafish. PLoS One. 2008:3(1):e1396.

44. Beck F. Homeobox genes in gut development. Gut. 2002;51(3):450-4.

45. Boland CR, Goel A. Microsatellite instability in colorectal cancer. Gastroenterology 2010;138(6):2073-87 e3.

46. Diaz LA Jr, Le DT. PD-1 blockade in tumors with mismatch-repair deficiency. N Engl J Med. 2015;373(20):1979.

47. Xiao Y, Freeman GJ. The microsatellite instable subset of colorectal cancer is a particularly good candidate for checkpoint blockade immunotherapy. Cancer Discov. 2015:5(1):16-8.

48. Shin US, Cho SS, Moon SM, Park SH, Jee SH, Jung EJ, et al. Is microsatellite instability really a good prognostic factor of colorectal cancer? Ann Coloproctol. 2014;30(1):28-34.

49. Earle JS, Luthra R, Romans A, Abraham R, Ensor J, Yao H, et al. Association of microRNA expression with microsatellite instability status in colorectal adenocarcinoma. J Mol Diagn. 2010;12(4):433-40.

50. Balaguer F, Moreira L, Lozano JJ, Link A, Ramirez G, Shen Y, et al. Colorectal cancers with microsatellite instability display unique miRNA profiles. Clin Cancer Res. 2011:17(19):6239-49.

51. Lanza G, Ferracin M, Gafa R, Veronese A, Spizzo R, Pichiorri F, et al. mRNA/ microRNA gene expression profile in microsatellite unstable colorectal cancer. Mol Cancer. 2007;6:54.

52. Tang S, Wu WK, Li X, Wong SH, Wong N, Chan MT, et al. Stratification of digestive cancers with different pathological features and survival outcomes by MicroRNA expression. Sci Rep. 2016;6:24466.

53. Sears $\mathrm{CL}$, Garrett WS. Microbes, microbiota, and colon cancer. Cell Host Microbe. 2014;15(3):317-28.

54. Waters LS, Storz G. Regulatory RNAs in bacteria. Cell. 2009;136(4):615-28.

55. Chen $\mathrm{H}$, Chen XZ, Waterboer T, Castro FA, Brenner H. Viral infections and colorectal cancer: a systematic review of epidemiological studies. Int J Cancer. 2015;137(1):12-24.

56. Butel JS. Viral carcinogenesis: revelation of molecular mechanisms and etiology of human disease. Carcinogenesis. 2000;21 (3):405-26.

57. Kuzembayeva M, Hayes M, Sugden B. Multiple functions are mediated by the miRNAs of Epstein-Barr virus. Curr Opin Virol. 2014;7:61-5.

58. Lo AK, To KF, Lo KW, Lung RW, Hui JW, Liao G, et al. Modulation of LMP1 protein expression by EBV-encoded microRNAs. Proc Natl Acad Sci U S A. 2007;104(41):16164-9.

59. Wong AM, Kong KL, Tsang JW, Kwong DL, Guan XY. Profiling of Epstein-Barr virus-encoded microRNAs in nasopharyngeal carcinoma reveals potential biomarkers and oncomirs. Cancer. 2012;118(3):698-710.

60. Najdi R, Holcombe RF, Waterman ML. Wnt signaling and colon carcinogenesis: beyond APC. J Carcinog. 2011;10:5. 
61. Ye Y, Zhou Y, Zhang L, Chen Y, Lyu X, Cai L, et al. EBV-miR-BART1 is involved in regulating metabolism-associated genes in nasopharyngeal carcinoma. Biochem Biophys Res Commun. 2013;436(1):19-24.

62. Vie N, Copois V, Bascoul-Mollevi C, Denis V, Bec N, Robert B, et al.

Overexpression of phosphoserine aminotransferase PSAT1 stimulates cell growth and increases chemoresistance of colon cancer cells. Mol Cancer. 2008;7:14.

63. Riley KJ, Rabinowitz GS, Yario TA, Luna JM, Darnell RB, Steitz JA. EBV and human microRNAs co-target oncogenic and apoptotic viral and human genes during latency. EMBO J. 2012;31(9):2207-21.

64. Cameron JE, Yin Q, Fewell C, Lacey M, McBride J, Wang X, et al. Epstein-Barr virus latent membrane protein 1 induces cellular MicroRNA miR-146a, a modulator of lymphocyte signaling pathways. J Virol. 2008;82(4):1946-58.

65. Ougolkov A, Zhang B, Yamashita K, Bilim V, Mai M, Fuchs SY, et al. Associations among beta-TrCP, an E3 ubiquitin ligase receptor, beta-catenin, and NF-kappaB in colorectal cancer. J Natl Cancer Inst. 2004;96(15):1161-70.

Ready to submit your research? Choose BMC and benefit from:

- fast, convenient online submission

- thorough peer review by experienced researchers in your field

- rapid publication on acceptance

- support for research data, including large and complex data types

- gold Open Access which fosters wider collaboration and increased citations

- maximum visibility for your research: over $100 \mathrm{M}$ website views per year

At $\mathrm{BMC}$, research is always in progress.

Learn more biomedcentral.com/submissions 